\title{
BIOACCUMULATION DE TROIS INSECTICIDES ORGANOCHLORES (LINDANE, DIELDRINE ET DDT) ET DES PCB CHEZ PLUSIEURS ESPECES DE FOURMIS [HYMENOPTERA -FORMICIDAE] EN BELGIQUE.
}

\author{
M.H. Debouge ('), J.P. Thome \& Ch. Jeuniaux \\ Université de Liège, Institut de Zoologie Ed. Van Beneden, \\ Laboratoire de Morphologie, Systématique et Ecologie animale, \\ Quai Van Beneden 22, B 4020 Liège, Belgique.
}

\begin{abstract}
L'étude de l'impact de 3 insecticides organochlorés (lindane, dielrine et DDT) et des PCB sur 5 espèces de fourmis abondamment représentées en Belgique montre qu'en région wallonne, la contamination des fourmis par ces toxiques est générale, mais reste faible quel que soit le milieu considéré. En moyenne, lordre de grandeur est de $20 \mathrm{ppb}$ pour le lindane, $4.4 \mathrm{ppb}$ pour la dieldrine, $13.4 \mathrm{ppb}$ pour le pp'DDE, 11.7 ppb pour l'op'DDD, 15,6 ppb pour le pp'DDE (3 métabolites du DDT) et de $200 \mathrm{ppb}$ pour les PCB. Les résultats obtenus ne montrent pas de relation entre le degré de contamination des ouvrières et celui du matériau de construction du nid. De plus, les concentrations en insecticides et en PCB des fourmis et des matériaux de construction des nids ne sont pas significativement différentes dans les milieux agricoles et forestiers.
\end{abstract}

MOTS CLES : Fourmis, Lindane, DDT, Dieldrine, PCB.

Les insecticides organochlorés et les PCB sont des substances toxiques peu biodégradables et très persistantes dans l'environnement. Ces composés sont d'autant plus dangereux qu'ils peuvent être l'objet d'une accumulation le long des chaînes trophiques, ce qui perturbe en définitive l'équilibre des écosystèmes. Parmi les insecticides organochlorés, le lindane est le seul qui soit encore abondamment utilisé aujourd'hui dans les pays d'Europe occidentale. D'autre part, par leur utilisation généralisée au niveau domestique et industriel, les PCB constituent également un risque important de contamination des écosystèmes. La persistance et la toxicité des PCB sont étroitement liées à leur teneur en chlore. Les isomères mono- et di-chlorés sont, en général, facilement dégradables, mais les isomères possédant plus de 4 atomes de chlore résistent mieux à la dégradation. La liposolubilité élevée de ces composés organochlorés, alliée à leur faible hydrosolubilité, en font des polluants qui s'accumulent dans les tissus de la plupart des organismes et qui sont difficilement métabolisés ou excrétés (Ullman, 1972 ; Hützinger et al., 1974 ; Portmann, 1979).

(l) Chercheur I.R.I.A. 
Si l'accumulation de ces substances fait l'objet de nombreux travaux chez les vertébrés et chez les animaux aquatiques, elle est par contre peu étudiée chez les invertébrés terrestres, notamment les insectes. Comme les fourmis jouent un róle important dans les écosystèmes terrestres, par les remaniements des sols et les transports de matériaux qu'elles réalisent, il était indispensable de chercher à connaitre l'impact de l'utilisation actuelle de ces composés organochlorés sur les populations de fourmis.

Le présent travail s’attache essentfellement à analyser le degré de bioaccumulation du lindane et des PCB chez plusieurs espèces de fourmis abondamment représentées en Belgique. En outre, nous avons voulu mesurer le degré de contamination de ces fourmis par dautres insecticides organochlorés (DDT et métabolites, dieldrine) actuellement interdits d'usage en Belgique mais toujours décelables dans les écosystèmes (Thomé \& Thomé, 1982).

\section{MATÉRIEL BIOLOGIQUE}

Une série de 134 échantillons ont été prélevés en milieux forestiers d'une part, en milieux agricoles d'autre part dans 2 régions situées à l'est de la Belgique (fig. 1). Cette étude porte sur 5 espèces de fourmis. Deux d'entre elles, Formica polyctena (Förster, 1850) et Formica rufa (L., 1758) font des nids en forme de dômes. On les retrouve uniquement en milieux forestiers (essentiellement en forèts d'épicéas). Trois autres espèces, Myrmica laevinodis (Nylander,

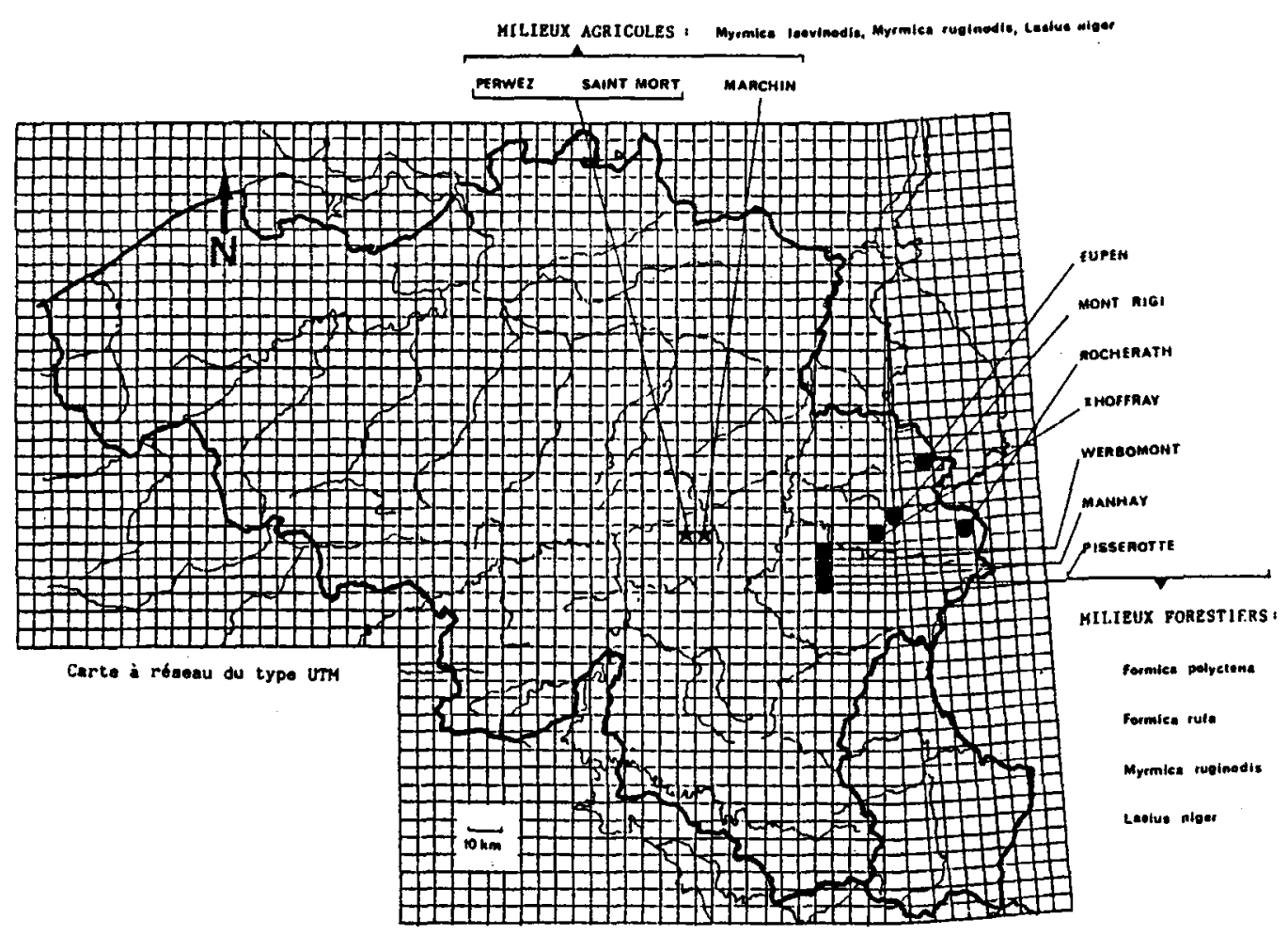

Fig. 1. Localisation des relevés en milieux forestiers $(\bullet)$ et en milieux agricoles $(\star)$. 
1846), Myrmica ruginodis (Nylander, 1846) et Lasius niger (L., 1758) font des nids dans la terre et sont présentes aussi bien en milieux forestiers qu'en milieux agricoles. La récolte des échantillons s'est faite manuellement. Dans chaque nid, nous avons prélevé un échantillon de fourmis (ouvrières), et un échantillon de matériau de construction du nid (aiguilles d'épicéas pour les Formica; terre pour les Myrmica et les Lasius). Les fourmis ont été triées, débarrassées de la terre ou autre matériau à l'aide d'un pinceau et plácées au congélateur $\left(-20^{\circ} \mathrm{C}\right)$ en attendant d'être analysées. Le matériau de construction des nids a également été pesé et congelé.

\section{MÉTHODES D'ANALYSE}

\section{EXTRACTION DES ORGANOCHLORÉS}

La méthode d'extraction utilisée est dérivée de celle de l'E.P.A. (1979) qui préconise l'utilisation d'acétonitrile suivie d'une partition liquide - liquide avec de l'hexane pour séparer les organochlorés des lipides. Lors des extractions, l'acétonitrile a été remplacé par l'acétone et la méthode ne comporte plus de séparation liquide - liquide, opération généralement longue.

A la sortie dù congélateur, les échantillons sont réchauffés à l'étuve à $30^{\circ} \mathrm{C}$. lls sont ensuite mélangés à 1 à $2 \mathrm{~g}$ de $\mathrm{Na}_{2} \mathrm{SO}_{4}$ anhydre, et broyés dans $5 \mathrm{ml}$ d'acétone à l'aide d'un homogéniseur de type «Turax». Les échantillons broyés sont agités à l'aide d'un homogéniseur de type «Vortex» pendant 2 minutes, puis centrifugès à $1750 \mathrm{G}$. Le surnageant est récupéré et le culot est à nouveau extrait par le mème procédé avec $5 \mathrm{ml}$ d'acétone. Les $10 \mathrm{ml}$ d'extrait acétonique sont évaporés à sec à l'aide d'un rotaévaporateur et l'extrait est repris dans $1 \mathrm{ml}$ d'hexane.

\section{Purification des EChantillons}

La purification des échantillons a été réalisée par une méthode de chromatographie liquide sur microcolonne de Florisil $\star$ (Waters Associates, Inc., Milford, MA', USA). Les colonnes de Florisil sont préparées en y faisant passer sous pression, successivement $2 \mathrm{ml}$ de diéthyléther, $2 \mathrm{ml}$ d'hexane diethylether $(50 / 50 ; \mathrm{V} / \mathrm{V})$ et $2 \mathrm{ml}$ d'hexane. Les extraits repris dans $1 \mathrm{ml}$ d'hexane sont déposés en tête de colonne. Les organochlorés sont élués à l'aide de 2 fois $2 \mathrm{ml}$ d'hexane. Les éluats sont recueillis ensemble. Les $5 \mathrm{ml}$ d'extrait recueillis sont concentrés sous flux d'azote jusqu'à un volume final de $1 \mathrm{ml}$.

\section{RENDEMENTS D'EXTRACTION ET DE PURIFICATION}

L'apport de 20 ng d'op'DDE, utilisé comme étalon interne, ajouté à l'échantillon avant le début de l'extraction, a permis de montrer que cette méthode donne d'excellents rendements d'extraction, proches de $100 \%$. En effet, la réalisation de 3 extractions successives d'un mème échantillon contenant une quantité connue d'étalon interne montre que lors de la $1^{\text {re }}$ extraction, la récupération est de $72.6 \% \pm 9.5$ d'op ${ }^{\prime} D D E, 20.6 \% \pm 8$ lors de la $2^{\mathfrak{e}}$ extraction, et $6.8 \% \pm 5$ lors de la $3^{\mathrm{C}}$ extraction. Cette expérience, répété à plusieurs reprises, montre que 2 extractions suffisent pour obtenir un rendement d'extraction proche de $100 \%$.

En outre, une série d'expériences préliminaires ont été réalisées à partir d'un mélange pur d'insecticides organochlorés et de PCB ( $50 \mathrm{pg}$ de chaque composé / $\mu$ d'hexane) ayant subi toutes les étapes d'extraction et de purification. Les rendements de récupération obtenus étaient voisins de $100 \%$ pour chacun des composés (lindane : $102 \% \pm 6$, dieldrine : $109 \% \pm 5$, DDT et métabolites : $100 \%( \pm 1, \mathrm{PCB}: 98 \%) \pm 3)$.

(*) cartouches Sep-Pack Florisil (granulométrie : 60-100 mesh ASTM ; degré d'activité : èlevé : humidité “ 2.5 \%) 


\section{DOSAGE DES ORGANOCHLORES}

Le dosage des pesticides organochlorés et des PCB a été réalisé par chromatographie en phase gazeuse à haute résolution. Deux $\mu 1$ d'extrait purifié sont analysés à l'aide d'un chromatographe Carlo Erba 4130 muni d'un détecteur à capture d'électrons ( $63 \mathrm{Ni}$ ). La colonne utilisée est une colonne capillaire verre WCOT ("wall coated open tubular») de $25 \mathrm{~m}$ de long et de $0.25 \mathrm{~mm}$ de diamètre interne. La phase stationnaire est du CPTM SIL 8 chimiquement liée et l'épaisseur du film de $0.11 \mu$ (Chrompack, Antwerpen, Belgique). Le système d'injection a été automatisé grâce à l'utilisation d'un injecteur d'échantillons solides Carlo Erba commandé par une vanne électropneumatique. Ce dispositif se place sur l'injecteur et permet l'analyse successive de 23 extraits contenus dans des capillaires de verre de $2.16 \mu l \pm 0.13$. Les capillaires sont préparés pour recevoir les échantillons par plusieurs rinçages successifs à l'hexane et à l'acétone. Après évaporation de ces solvants, sous flux d'azote, la paroi interne des capillaires est recouverte de phase stationnaire SE-30 de la façon suivante : une solution à $2 \%$ de SE-30 dans l'hexane est introduite par capillarité. Après évaporation du solvant, les capillaires sont prêts à recevoir les extraits. Les extraits sont introduits dans les capillaires à l'aide d'une micro-seringue (Halminton $5 \mu 1$ ). Après évaporation du solvant, les capillaires sont disposés dans le barillet de l'injecteur automatique réfrigéré à $0^{\circ} \mathrm{C}$ de façon à éviter les pertes par évaporation.

\section{CALCUL DES CONCENTRATIONS}

Les concentrations d'insecticides organochlorés et de PCB dans les échantillons ont été calculées par rapport à l'étalon interne (op'DDE) et sont exprimées en $\mathrm{ng} / \mathrm{g}$ de poids frais (ppb). Les concentrations de PCB totaux sont exprimées sous forme d'Aroclor 1260. La quantification a été réalisée en se basant sur les 9 pics les plus importants d'un standard de ce mélange de PCB. Les 9 pics correspondent aux congénères et isomères repris dans ce tableau 1.

L'identité des PCB et des insecticides organochlorés étudiés a été vérifiée par spectrométrie de masse dans quelques échantillons. La présence des insecticides et/ou des PCB a été confirmée dans tous les cas.

TABleau 1

Numéro de Balschmitter, congénères et isomères des 9 pics les plus importants d'un mélange de PCB sous forme d'Aroclor 1260

\begin{tabular}{cc}
\hline $\begin{array}{c}\text { Numéro de Balschmitter } \\
\text { (Balschmitter, 1980) }\end{array}$ & $\begin{array}{c}\text { Congénères } \\
\text { et isomeres }\end{array}$ \\
\hline 149 & $22^{\prime} 3^{\prime} 456^{\prime}$ \\
153 & $22^{\prime} 44^{\prime} 55^{\prime}$ \\
151 & $22^{\prime} 355^{\prime} 6^{\prime}$ \\
138 & $22^{\prime} 344^{\prime} 5^{\prime}$ \\
187 & $22^{\prime} 34^{\prime} 55^{\prime} 6$ \\
174 & $22^{\prime} 33^{\prime} 45^{\prime} 6$ \\
180 & $22^{\prime} 344^{\prime} 55^{\prime}$ \\
170 & $22^{3} 33^{\prime} 44^{\prime} 5^{\prime}$ \\
196 & $22^{\prime} 33^{\prime} 44^{\prime} 56^{\prime}$ \\
\hline
\end{tabular}




\section{RÉSULTATS ET DISCUSSION}

L'ensemble des résultats montre que les fourmis des milieux étudiés de l'est de la Belgique sont plus particulièrement contaminées par 2 types de composés organochlorés : le lindane et les PCB. Examinons simultanément les résultats concernant ces 2 groupes de substances, avant d'examiner ceux qui concernent les autres composés chlorés.

\section{CONTAMINATION PAR LE LINDANE ET LES PCB}

Les figures 2 et 3 représentent respectivement les relations existant entre les concentrations en PCB et en lindane décelées dans les ouvrières et dans le matériau de construction des nids pour chacun des groupes d'un même genre de fourmis. Ces figures montrent l'absence de relation directe significative entre le degré de contamination des ouvrières et celui du matériau de construction des nids, et montrent l'absence de différence significative entre les 3 groupes d'espèces de fourmis.

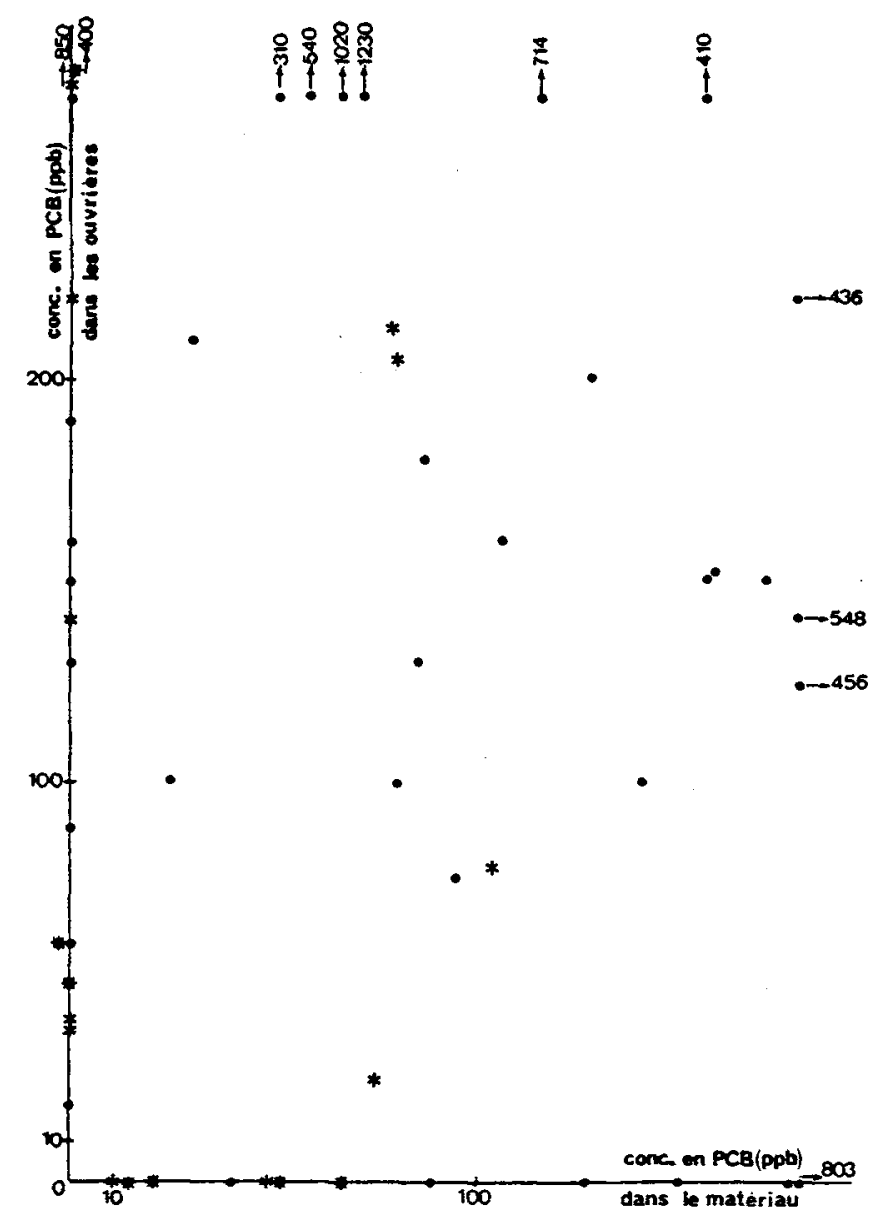

Fig. 2. Relation entre les concentrations en PCB dans les ouvrières des groupes d'espèces Formica (๑), Myrmica (*), Lasius (*) et dans le matériau de construction des nids de ces mêmes fourmis. 


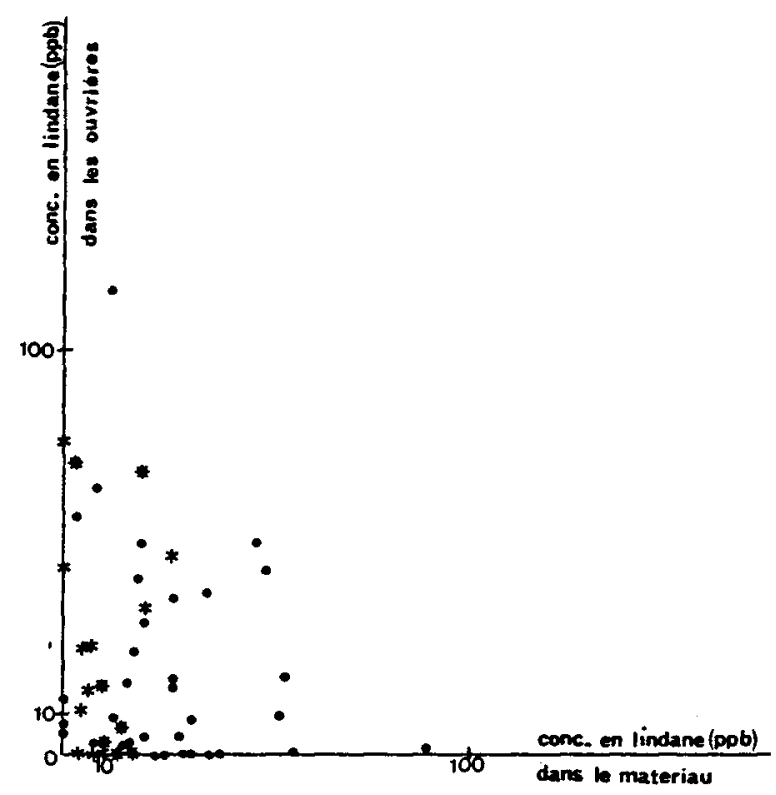

Fig. 3. Relation entre les concentrations en lindane dans les ouvrières des groupes d'espèces Formica (๑), Myrmica $(*)$, Lasius (*) et dans le matériau de construction des nids de ces mêmes fourmis.

Dans les figures 4 à 7 les concentrations moyennes $(\bar{x})$ en lindane et en PCB (et leurs écarts types $(\sigma))$ ont été calculées tant pour les ouvrières, toutes espèces confondues, que pour le matériau de construction des nids de fourmis étudiées, pour chacune des localités prospectées et pour chaque type de milieu étudié (milieux agricoles et forestiers).

Les figures 4 et 5 montrent que la contamination par le lindane est aussi importante en milieux forestiers qu'en milieux agricoles. En milieux agricoles, cette contamination peut s'expliquer par le fait que tous les relevés ont été réalisés aux abords de champs cultivés pulvérisés par du GAMMA-COL (1) (suspension contenant $80 \%$ de lindane) juste avant chaque semis de betteraves (en principe, plusieurs pulvérisations successives une fois tous les trois ans). En milieux forestiers, une préparation à base de lindane est utilisée sous forme de poudre répandue autour des pieds des jeunes épicéas ou sous forme d'une solution pulvérisée autour des jeunes plants (SYLVOGAM : $3.75 \%$ de lindane) (2). L'utilisation du lindane en pessière est donc localisée mais peut toutefois expliquer en partie la présence de cet insecticide dans la plupart de nos échantillons. En outre, le lindane, ayant une tension de vapeur élevée, pourrait ètre transporté par les vents dominants d'ouest, des régions agricoles, où il est encore utilisé, vers les régions forestières, situées à l'est des zones agricoles et où nous avons fait nos relevés.

Les figures 6 et 7 montrent que les concentrations moyennes en PCB dans les ouvrières ainsi que dans le matériau de construction des nids sont fort semblables en milieux forestiers et en milieux agricoles. Dans les écosystèmes terrestres, le transport atmosphérique semble être la voie principale d'entrée des PCB et des insecticides organochlorés dans l'environnement. Ils sont transportés soit, sous forme d'aérosols, soit adsorbés par des particules. Ces toxiques contribuent donc à la contamination des écosystèmes à l'occasion de retombées

(1) GAMMA-COL (n' d'agrégation 6210) est une marque déposée d'l.C.I.

(2) SYLVOGAM ( ${ }^{\circ}$ d'agrégation 1721 ) est une marque deposée d'I.C.I. 




Fig. 4. Concentrations moyennes (ppb) en lindane dans les fourmis ouvrières toutes espèces confondues (à gauche) et dans le matériau de construction des nids (à droite) en milieux forestiers. Les barres verticales représentent les écarts types (partie positive).

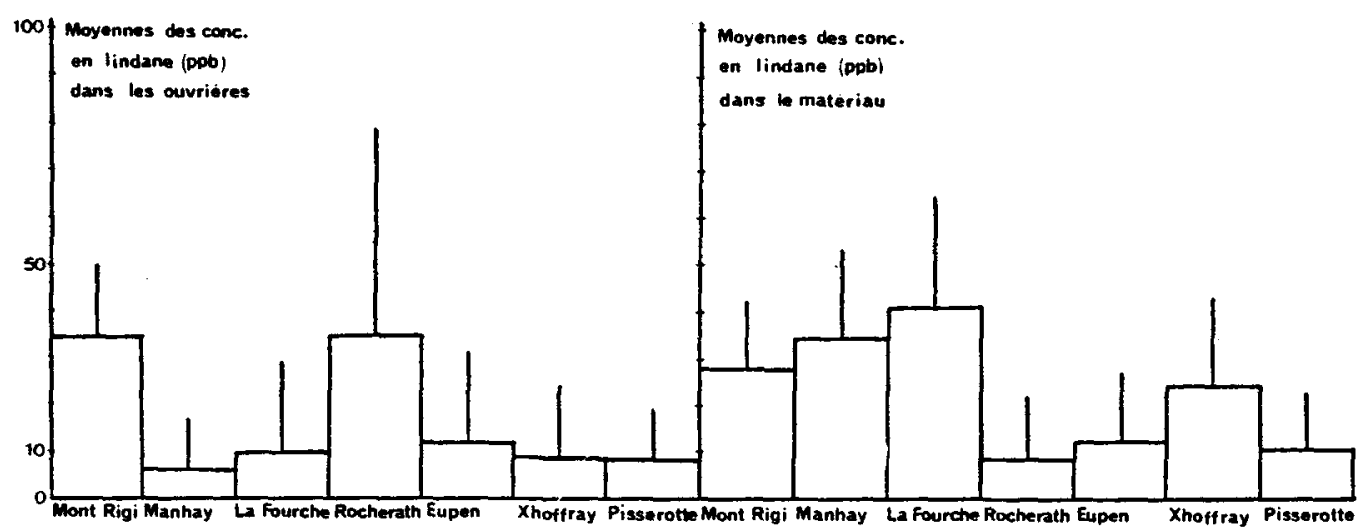

Fig. 5. Concentrations moyennes (ppb) en lindane dans les fourmis ouvrières. toutes espèces confondues (à gauche) et dans le matériau de construction des nids (à droite) en milieux agricoles. Les barres verticales représentent les écarts types (partie positive).

particulaires et des précipitations atmosphériques (Nisbet \& Sarofim, 1972 ; Lafontaine \& Delforge, 1979). Les PCB passant facilement dans l'atmosphère sous forme de particules, par effet aérosol, seraient entrainés par les vents dominants des zones industrielles où ces substances sont abondamment employées (vallée de la Meuse) vers nos stations forestières de récoltes d'échantillons, toutes situées à l'est du sillon Sambre et Meuse.

\section{CONTAMINATION PAR LA DIELDRINE ET LE DDT}

Les résultats repris dans le tableau 2 montrent clairement que dans beaucoup de localités, on retrouve des traces de dieldrine et de DDT. Le DDT ne se retrouve pas en tant que tel, mais sous la forme de ses métabolites. Trois d'entre eux ont été décelés dans les échantillons, il s'agit du pp'DDE, de l'op'DDD et du pp'DDD. Les concentrations décelées sont tout à fait comparables à celles de la dieldrine (tableau 2). 


\section{TABLEAU 2}

Moyennes $(\bar{x})$ et écarts types $(\sigma)$ des concentrations en dieldrine et en métabolites du DDT dans les fourmis ouvrières, toutes espèces confondues et dans le matériau de construction des nids (les concentrations sont exprimées en ppb).

\begin{tabular}{lcccccccc}
\hline & \multicolumn{1}{c}{ dieldrine } & pp'DDE & op'DDD & \multicolumn{2}{c}{ pp'DDD } \\
\hline & $\overline{\mathrm{x}}$ & $\sigma$ & $\overline{\mathrm{x}}$ & $\sigma$ & $\overline{\mathrm{x}}$ & $\sigma$ & $\overline{\mathrm{x}}$ & $\sigma$ \\
\hline ouvrières & 4.4 & 10.2 & 13.4 & 36.5 & 11.7 & 18.2 & 15.6 & 29.2 \\
matériau & 10.9 & 22 & 5.9 & 8.2 & 9 & 17 & 5.7 & 9.5 \\
\hline
\end{tabular}

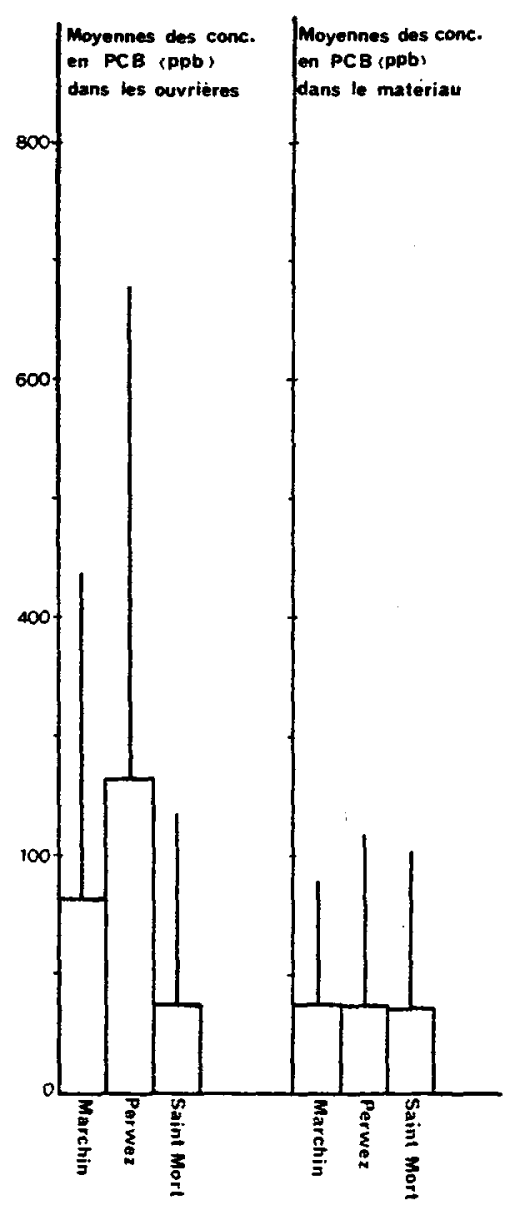

Fig. 6. Concentrations moyennes (ppb) en PCB dans les fourmis ouvrières, toutes espèces confondues (à gauche) et dans le materiau de construction des nids (à droite) en milieux forestiers. Les barres verticales representent les écarts types (partie positive). 


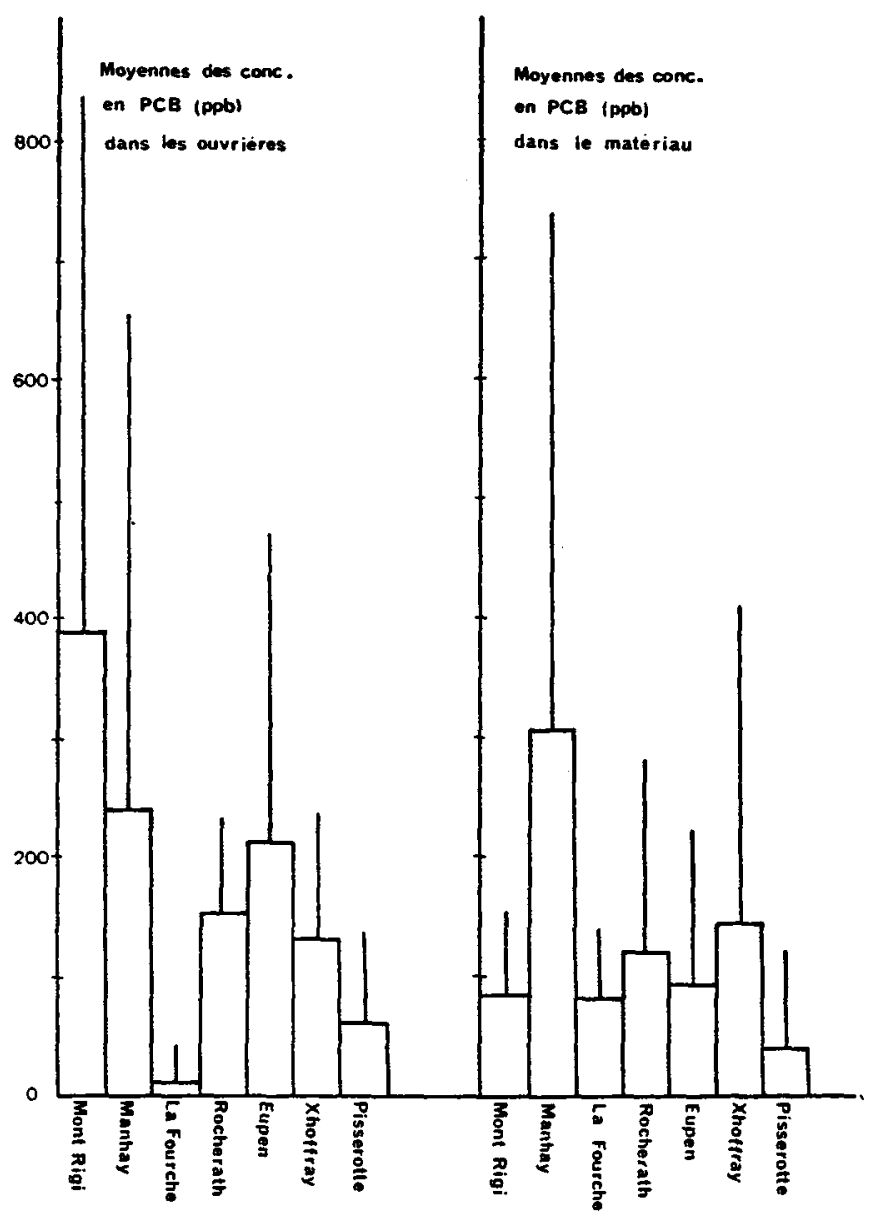

Fig. 7. Concentrations moyennes (ppb) en PCB dans les fourmis ouvrières, toutes especes confondues (à gauche) et dans le matériau de construction des nids (ä droite) en milieux agricoles. Les barres verticales représentent les écarts types (partie positive).

\section{CONCLUSIONS}

Les degrés de contamination des 5 espèces de fourmis étudiées (Formica rufa, Formica polyctena, Myrmica ruginodis, Myrmica laevinodis et Lasius niger) ne sont pas significativement différents et les différentes espèces ont, de ce fait, èté considérées sans distinction. En région wallonne, la contamination par le lindane et les PCB est générale, mais elle reste faible quel que soit le milieu considéré. En moyenne, l'ordre de grandeur est de $20 \mathrm{ppb}$ pour le lindane et de $200 \mathrm{ppb}$ pour les PCB. Il ne semble pas exister de relations entre le degré de contamination des ouvrières et celui du matériau de construction des nids tant pour le lindane que pour les PCB. Nos résultats suggèrent également l'absence de relation entre le degré de contamination des ouvrières, celui du matériau de construction des nids et le type de milieu étudié. Les échantillons relevés en milieux agricoles (cultures de betteraves) présentent des concentrations en lindane et en PCB fort semblables à celles des échantillons relevés en milieux forestiers. 
La contamination des milieux ne résulte donc pas directement et uniquement de traitemènts directs par le lindane ou de contamination directe par les PCB. Ces toxiques se propagent vraisemblablement par transport atmosphérique de particules et peuvent, de ce fait, être emportés très loin des sites d'application et d'utilisation, surtout dans la direction des vents dominants, en l'occurrence vers l'est de la Belgique.

Les teneurs des autres insecticides organochlorés (dieldrine et DDT) décelées tant dans les fourmis que dans le matériau de construction des nids sont nettement moins élevées que les concentrations en lindane et en PCB. Cette situation résulte inévitablement de linterdiction d'utilisation de la plupart des insecticides organochlorés (à l'exception du lindane) imposée entre 1974 et 1976 en Belgique. Cependant, malgré le retrait du marché officiel, ces organochlorés continuent à assurer une contamination généralisée de la faune myrmécologique.

\section{SUMMARY}

Bioaccumulation of three organochlorinated insecticides (lindane, dieldrin and DDT) and PCB in several species of ants [Hymenoptera-Formicidae] in Belgium.

The impact of 3 organochlorinated insecticides (lindane, dieldrin and DDT) and of PCB on 5 ant species in Belgium has been estimated. Our study has clearly shown that the contamination of ants by these toxics was generalized. However, the level of intoxication was relatively low and the mean concentrations were around $20 \mathrm{ppb}$ of lindane, 4.4. ppb of dieldrin, $13.4 \mathrm{ppb}$ of pp'DDE, $11.7 \mathrm{ppb}$ of op'DDD, $15.6 \mathrm{ppb}$ of pp'DDD (3 metabolites of DDT) and $200 \mathrm{ppb}$ of PCB. There was no significative relation between the degree of contamination of ants, on one hand, and of nest material, on the other hand. Moreover, the concentrations of insectides and PCB residues were not significatively different in agricultural and forested areas.

KEY-WORDS : ants, lindane, D.D.T., dieldrin, PCB.

Reçu le 12 juin 1986 ; Accepté le 24 novembre 1986.

\section{REFERENCES}

Ballschmiter, K. \& Zell, M. - 1980. Analysis of Polychlorinated Biphenyls (PCB) by Glass Capillary Gas Chromatography. Composition of Technical Aroclor and Clophen - PCB Mixtures. Fresenius $Z$. Anal. Chem., 302, 20-31.

E.P.A. - 1979. Manual of analytical methods for the analysis of pesticides in human and environmental samples. (R.R. Watt). - EPA - 600/8-80-038.

Gaspar, C. - 1966. Les fourmis et lagriculture [Hymenoptera : Formicidae] - Ann. Gembloux, 72, 235-243.

Gaspar, C. - 1971. Les fourmis de la Famenne (une étude zoogéographique). - Bull. Inst. R. Sci. Nat. Belg., 47 (20), 109 p.

Hutzinger, O, Safe, S. \& Zitko, V. - 1974. The chemistry of PCB's. - CRC Press Inc., Boca Raton, 269 p.

Lafontaine, A. \& Delforge-Lefebvre, S. - 1979. Evaaluation de l'impact des PCB et PCT sur l'environnement aquatique. - CCE Environ., 1.78-80, 177 p.

Nisbet, C.T. \& Sarofim, A.F. - 1972. Rates and routes of transport of PCB's in the environment. Environ. Hlth. Persp., 1, $21 \mathrm{p}$.

Portman, J.E. - 1979. Evaluation of the impact on the aquatic environment of HCB isomers, HCB, DDT (+ DDE and DDD), heptachlor (+ heptachlor epoxide) and chlordane. - CEE Environ., $488,79,337 \mathrm{p}$.

Rose, J.A. - 1971. In : Matsumura, F. - 1975. Toxicology of insecticides. Plenum Press., New York and London, $503 \mathrm{p}$. 
Thome, J.P. \& Thome, M. - 1982. Enquête sur les espèces de vertébrés menacés de disparition en Wallonnie. VII : Les pesticides et les métaux lourds comme facteur de risque pour la faune sauvage. - Ministère de la région wallonne pour l'eau. l'environnement et la vie rurale. Dépót légal $\mathrm{D} / 1982 / 3730 / 1$.

UImann, E. - 1972. Lindane, monographie d'un insecticide. (Schillinger, K., ed.), Verlag. Freiburg im Breisgau. 383 p.

Van Daele, \& Thome, J.P. - 1986. Purification of PCB Contaminated water by Chitosan : a Biological Test of Efficiency using the Common Barbel (Barbus barbus). - Bull. Environ. Contam. Toxicol., $37 ; 858-865$. 